\title{
Low-lying excitations at the rare-earth site due to the rattling motion in the filled skutterudite $\mathrm{LaOs}_{4} \mathrm{Sb}_{12}$ revealed by ${ }^{139} \mathrm{La} \mathrm{NMR}$ and ${ }^{121 / 123} \mathrm{Sb} \mathrm{NQR}$
}

\author{
Yusuke Nakai, ${ }^{1}$, 田 Kenji Ishida, ${ }^{1}$ Hitoshi Sugawara, ${ }^{2}$ Daisuke Kikuchi, ${ }^{3}$ and Hideyuki Sato ${ }^{3}$ \\ ${ }^{1}$ Department of Physics, Graduate School of Science, Kyoto University, Kyoto 606-8502, Japan, \\ ${ }^{2}$ Faculty of Integrated Arts and Sciences, University of Tokushima, Tokushima 770-8502, Japan, \\ ${ }^{3}$ Graduate School of Science, Tokyo Metropolitan University, Hachioji, Tokyo 192-0397, Japan
}

(Dated: October 28, 2018)

\begin{abstract}
We report experimental results of nuclear magnetic resonance (NMR) at the La site and nuclear quadrupole resonance (NQR) at the Sb site in the filled skutterudite $\mathrm{LaOs}_{4} \mathrm{Sb}_{12}$. We found that the nuclear spin-lattice relaxation rate divided by temperature $1 / T_{1} T$ at the La site exhibits a different temperature dependence from that at the $\mathrm{Sb}$ site. Although $1 / T_{1} T$ at the $\mathrm{Sb}$ site is explained by the Korringa mechanism, $1 / T_{1} T$ at the La site exhibits a broad maximum around $50 \mathrm{~K}$, showing the presence of an additional contribution at the La site. The additional low-lying excitations observed at the La site can be understood with the relaxation from anharmonic phonons due to the rattling motion of the La atoms.

PACS numbers: 71.27.+a, 74.25.Kc, 76.60.-k, 76.60.Gv
\end{abstract}

The filled skutterudite compound $R T_{4} X_{12}$ ( $R=$ rare earth; $T=\mathrm{Fe}, \mathrm{Ru}, \mathrm{Os} ; X=\mathrm{P}, \mathrm{As}, \mathrm{Sb})$ has a unique structure in which the $R$ ion is located in an oversized cage made of $12 X$ atoms. The $R$ ion is loosely bound inside of the $X_{12}$ cage because the cage size is much larger than the $R$ ion. A weak atomic interaction between the $R$ ion and the cage atom is considered to cause the vibration of the $R$ ion to be highly anharmonic. Due to this cage structure, the anharmonic vibration with large amplitude of the $R$ ion in the cage occurs, which is called grattling.h From a practical point of view, the rattling attracts much attention because it may reduce thermal conductivity, which is a key to producing high-performance thermoelectric materials $\underline{\underline{1}}$ The low thermal conductivity is believed to result from localized vibrational modes at low energy, attributed to the rattling ${ }_{2}^{2}$ Specific heat results suggest a nearly dispersionless low-energy optical mode that is characterized by an Einstein phonon mode $\frac{3}{3} \mathrm{~A}$ large Debye- Waller factor obtained with neutron diffraction experiments indicates a large thermal displacement of the $R$ ion. $\underline{\underline{4}}$ In Raman spectra, a second-order phonon peak appears originating from an almost dispersionless optical mode at low energy relevant to the rattling, 5,6 An anomalous Debye-type dispersion of elastic constants found in ultrasound measurements is also attributable to the rattling $\underline{\underline{?}}$

Recently, it has been recognized that the rattling motion might be a key phenomenon to understand the exotic heavyfermion ( $\mathrm{HF})$ state in $R \mathrm{Os}_{4} \mathrm{Sb}_{12}(R=\mathrm{Pr}, \mathrm{Sm}$, $\mathrm{Nd})$. The exotic HF state shows very different behavior from magnetic Kondo materials such as Ce- or Ubased HF compounds. They do not exhibit $-\log T$ behavior in the resistivity. $.9 .10,11$ Furthermore, a large electronic specific-heat coefficient $\left[\gamma=0.82 \mathrm{~J} /\left(\mathrm{K}^{2} \mathrm{~mol}\right)\right]$ in $\mathrm{SmOs}_{4} \mathrm{Sb}_{12}$ is insensitive to magnetic fields $\stackrel{\underline{9}}{\underline{9}}$ These results suggest that the exotic HF state results from a nonmagnetic origin. This contrasts with the conventional HF state formed by magnetic interactions between the conduction electron and localized $f$ electron $(c-f$ inter- action). In this situation, the rattling motion of the $R$ ion is invoked to be related to the formation of the exotic HF state. In fact, HF states originating from anharmonic ion vibrations have been analyzed theoretically: ${ }^{12,13}$

Characterizing the rattling motion is quite important to understand the low thermal conductivity and the exotic HF state. However, the characterization is difficult from bulk measurements because the rattling is localized vibrational modes at the rare-earth site. In general, one can extract the localized vibrational modes through comparing between a filled skutterudite and an unfilled one. However, such a comparison is impossible when an unfilled skutterudite cannot be synthesized or is not appropriate when filling $R$ ions change the electronic state significantly. For these reasons, it is desirable to compare physical properties at the $R$ site with those at the Sb site to characterize the rattling in the same compound. For this purpose, nuclear magnetic resonance (NMR) and nuclear quadrupole resonance (NQR) are suitable probes, because NMR and NQR can derive low-lying excitations site-selectively due to the rattling motion. Although it is desirable to perform Pr or Sm NMR measurements, these nuclei are too magnetic to allow NMR measurements. For this reason, we instead performed ${ }^{139} \mathrm{La}$ NMR and ${ }^{121 / 123} \mathrm{Sb}$ NQR experiments on $\mathrm{LaOs}_{4} \mathrm{Sb}_{12}$ because the characteristic motion of $R$ ions is believed to be similar for the $R \mathrm{Os}_{4} \mathrm{Sb}_{12}(R=\mathrm{La}, \mathrm{Pr}, \mathrm{Sm}, \mathrm{Nd})$ family. Because the ${ }^{139} \mathrm{La}$ nucleus $(I=7 / 2)$ has an electric quadrupolar moment $Q$, the nuclear spin-lattice relaxation rate $1 / T_{1}$ at the La site can directly probe the unusual dynamics of the La ions through quadrupolar coupling of the La nucleus to the electric field gradient (EFG).

In this paper, we report results of ${ }^{139} \mathrm{La} \mathrm{NMR}$ and $121 / 123 \mathrm{Sb}$ NQR measurements on $\mathrm{LaOs}_{4} \mathrm{Sb}_{12}$. We found that $1 / T_{1} T$ at the La site exhibits a different temperature dependence from that at the Sb site. By subtracting the conduction electron contribution from the observed $1 / T_{1} T$ at the La site, we obtained additional relaxation at the La site ${ }^{139}\left(1 / T_{1} T\right)_{\text {add }}{ }^{139}\left(1 / T_{1} T\right)_{\text {add }}$ is nearly con- 


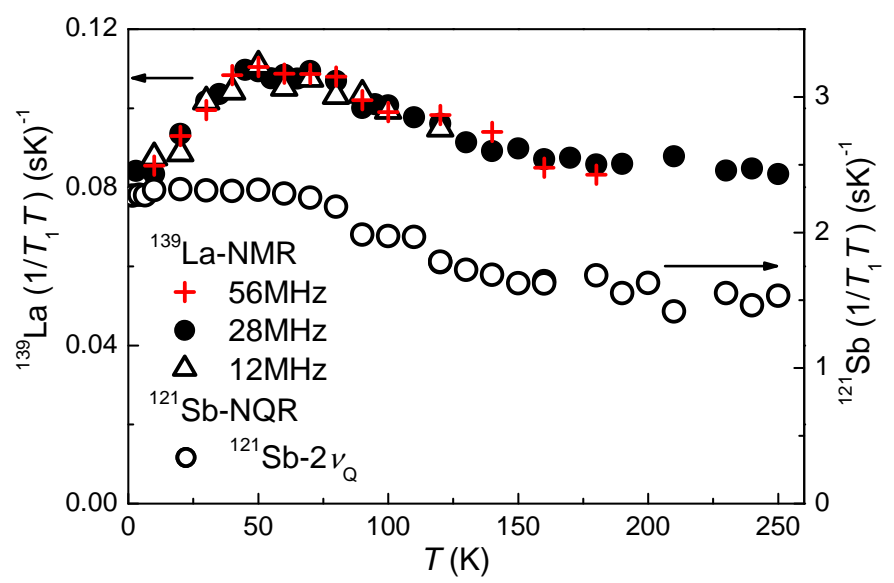

FIG. 1: (Color online) Temperature dependence of $1 / T_{1} T$ at the La and $\mathrm{Sb}$ site under various magnetic fields for La NMR. The broad maximum is found around $50 \mathrm{~K}$ only at the La site.

stant at high temperatures and decreases rapidly below about $50 \mathrm{~K}$. We show that this temperature dependence can be explained quantitatively by a recent theoretical calculation based on the Raman process of anharmonic phonons due to the rattling motion. $\stackrel{14}{\underline{1}}$ From the present result, we suggest that the low-lying excitations caused by the rattling of $R$ ions might be important to understand the exotic HF state in $R \mathrm{Os}_{4} \mathrm{Sb}_{12}(R=\mathrm{Pr}, \mathrm{Sm}, \mathrm{Nd})$.

Single crystals of $\mathrm{LaOs}_{4} \mathrm{Sb}_{12}$ were grown with the Sbflux method 15 and were powdered for NMR and NQR measurements. The observation of de Haas-van Alphen oscillations demonstrates that we were using a highquality sample $\stackrel{15}{\underline{1}} \mathrm{NMR}$ and NQR measurements were performed using a conventional pulsed spectrometer in the temperature range of $T=0.6 !-! 250 \mathrm{~K}$ using a ${ }^{3} \mathrm{He}-$ ${ }^{4} \mathrm{He}$-dilution refrigerator. The nuclear spin-lattice relaxation rate $1 / T_{1}$ of La nuclei was measured using the saturation recovery method and was uniquely determined with a single component. $1 / T_{1}$ of ${ }^{121} \mathrm{Sb}\left({ }^{123} \mathrm{Sb}\right) \mathrm{NQR}$ was measured around $84(78) \mathrm{MHz}$ using a $2 \nu_{Q}\left(3 \nu_{Q}\right)$ transition. $\underline{16}$ The magnetic susceptibility $\chi$ was measured for the powdered sample with a superconducting quantum interference device (SQUID) magnetometer (Quantum Design MPMS).

Figure 1 shows the temperature dependence of $1 / T_{1} T$ at the La site under various magnetic fields, along with that at the ${ }^{121} \mathrm{Sb}$ site (open circles). ${ }^{121}\left(1 / T_{1} T\right)$ is consistent with the previous report 17 We found that $1 / T_{1} T$ at the $\mathrm{La}$ and $\mathrm{Sb}$ sites exhibits different $T$ dependences; the broad maximum around $50 \mathrm{~K}$ appears only at the La site. This is a marked contrast to the La-based skutterudite $\mathrm{LaFe}_{4} \mathrm{P}_{12}$, in which $1 / T_{1}$ at both La and $\mathrm{P}$ sites exhibits the same temperature dependence, $\underline{\underline{18}, \underline{19}}$

To clarify the origin of the site-dependent $1 / T_{1} T$, we measured the bulk susceptibility $\chi$ and the Knight shift at the La site $\left({ }^{139} K\right)$ as shown in Fig. 2. From a good linear relation between ${ }^{139} \mathrm{~K}$ and $\chi$, the temperature dependence of $\chi$ is not affected by impurity phases, but is

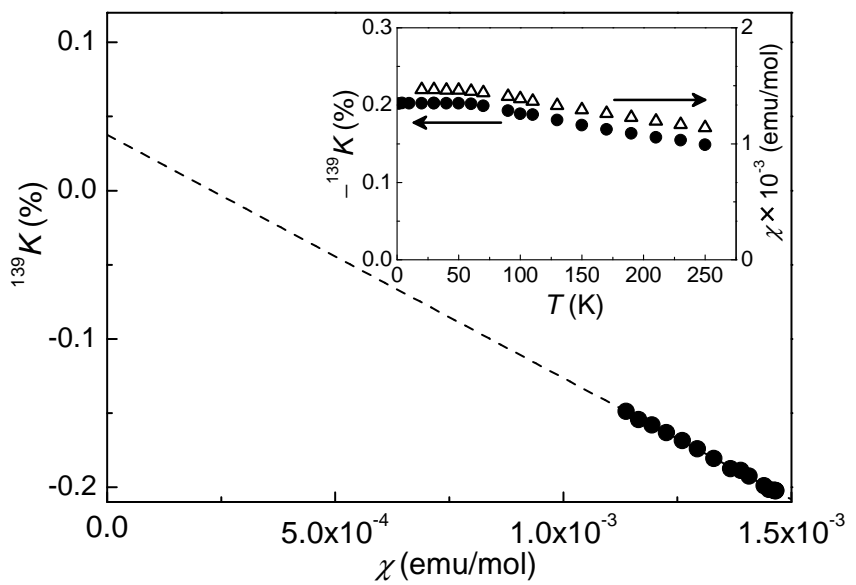

FIG. 2: The ${ }^{139}$ La Knight shift ${ }^{139} K$ is plotted against the bulk susceptibility $\chi$. The hyperfine coupling constant at the La site is estimated to be $-10.4 \mathrm{kOe} / \mu_{B}$. Inset: Temperature dependence of $\chi$ and ${ }^{139} K$.

intrinsic behavior of $\mathrm{LaOs}_{4} \mathrm{Sb}_{12}$. It is shown that $1 / T_{1} T$ at the $\mathrm{Sb}$ site exhibits a temperature dependence similar to $\chi^{2}$, but $1 / T_{1} T$ at the La site does not. In fact, ${ }^{121}\left(1 / T_{1} T\right)$ is scaled with $\chi^{2}$ as shown in Fig. 3. Furthermore, the ${ }^{121 / 123} \mathrm{Sb}$-isotopic ratio of $1 / T_{1}$ in the whole temperature range is consistent with the case of magnetic relaxations $\left(=\left({ }^{121} \gamma /{ }^{123} \gamma\right)^{2}=3.4\right)$ as shown in the inset of Fig. 3. These results indicate that $1 / T_{1} T$ at the $\mathrm{Sb}$ site is governed by magnetic fluctuations, which are related to the static susceptibility through the Korringa mechanism. Note that the temperature dependence of $1 / T_{1} T$ at the $\mathrm{Sb}$ site is considered to originate from narrow Os- $5 d$ bands, which is suggested by band calculations $\frac{20}{\underline{O}}$ Then, it seems natural to consider that the broad peak of $1 / T_{1} T$ at the La site is ascribed to additional low-lying excitations at the La site. The field-independent $1 / T_{1} T$ (see Fig. 1) implies that the lowlying excitations arise from nonmagnetic origin.

Next, we analyze the low-lying excitations using the temperature dependence of $1 / T_{1} T$ at the La site. We consider that $1 / T_{1} T$ at the La site is governed not only by the Korringa mechanism but also by local fluctuations of the EFG due to the rattling motion of the La ions. In fact, ultrasonic dispersion is found around 50

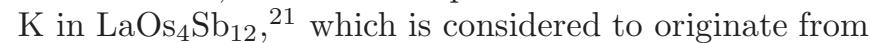
the rattling motion of the La atoms as in $\mathrm{PrOs}_{4} \mathrm{Sb}_{12}{ }^{\underline{7}}$ We assume that the fluctuations of the EFG disappear at low temperatures and that the Korringa mechanism dominates the relaxation at the La site at low temperatures, because $1 / T_{1} T$ at the La sites becomes constant below $10 \mathrm{~K}$ down to $0.6 \mathrm{~K}$ as $1 / T_{1} T$ of ${ }^{121} \mathrm{Sb}$ does as shown in the inset of Fig. 4. To estimate the contribution from the Korringa law at the La site, we normalized $1 / T_{1} T$ of ${ }^{121} \mathrm{Sb}$ to make it equal to that of La below 10 $\mathrm{K}$, as shown in the inset of Fig. 4 . Then, we obtained the additional fluctuations $\left(1 / T_{1} T\right)_{\text {add }}$, which is considered to arise from EFG fluctuations related to the rattling, as shown in Fig. 4: nearly constant at high temperatures 


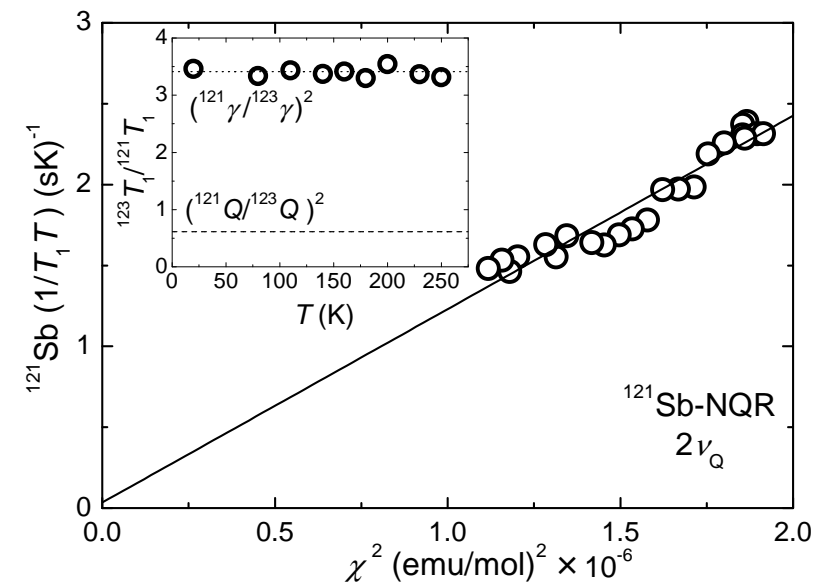

FIG. 3: Plot of $1 / T_{1} T$ of Sb against $\chi$ squared. The Korringa relaxation mechanism governs $1 / T_{1} T$ at the $\mathrm{Sb}$ site. Inset: the isotropic ratio of $1 / T_{1}$ for the $\mathrm{Sb}$ nucleus. The dotted (dashed) line shows the squared ratio of the nuclear magnetic (quadrupolar) moments.

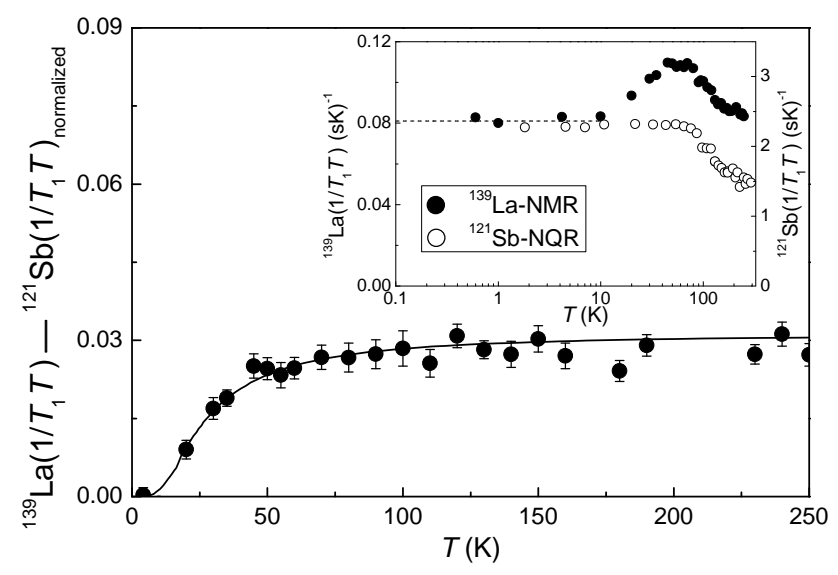

FIG. 4: The difference between $1 / T_{1} T$ of La and normalized $1 / T_{1} T$ of ${ }^{121} \mathrm{Sb}$, which is considered to arise from the rattling motion of the La ions. The solid line is the calculated result cited from Ref. $14\left[\beta=2.0, \omega_{r}(T=0)=60 \mathrm{~K}\right]$, which is consistent with the experimental data.

and decreasing rapidly below $50 \mathrm{~K}$. Nuclear spin relaxation due to phonons is very rare in metals, but the small value of $\left(1 / T_{1} T\right)_{\text {add }}\left(\sim 0.03 \mathrm{~s}^{-1} \mathrm{~K}^{-1}\right)$ can be explained in terms of phononic relaxation. In fact, recent observations of NMR relaxation rates at the $\mathrm{K}$ site in the $\beta$-pyrochlore superconductor $\mathrm{KOs}_{2} \mathrm{O}_{6}$ have been demonstrated to be entirely dominated by the vibration of the $\mathrm{K}$ ion via coupling of the EFG to the nuclear quadrupole moment 22 The phononic contribution at the ${ }^{41} \mathrm{~K}$ site in $\mathrm{KO}_{2} \mathrm{O}_{6}$ is $1 / T_{1} T \sim 0.009 \mathrm{~s}^{-1} \mathrm{~K}^{-1}$, which is the same order of magnitude as $\left(1 / T_{1} T\right)_{\text {add }}$ at the La site in $\mathrm{LaOs}_{4} \mathrm{Sb}_{12}$. However, the EFG fluctuations of the La atom are not the usual harmonic phonons. If one assumes NMR relaxation from the Raman process of harmonic phonons, the obtained temperature dependence of $\left(1 / T_{1} T\right)_{\text {add }}$ cannot be explained: $1 / T_{1} T$ is proportional to $T$ at high temperatures in the Raman process for harmonic phonons $\underline{23}$
The inconsistency invokes the need to take into account the anharmonicity that is one of the important characteristics of the rattling motion.

Quite recently, Dahm and Ueda calculated NMR relaxation due to coupling to a strongly anharmonic and damped phonon mode, and explained successfully the temperature dependence of $1 / T_{1}$ at the $\mathrm{K}$ site in

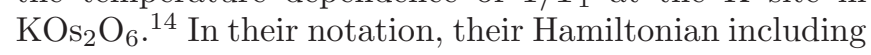
anharmonic term is described as follows:

$$
H=\frac{p^{2}}{2 M}+\frac{1}{2} a x^{2}+\frac{1}{4} b x^{4},
$$

They treat this Hamiltonian in a self-consistent quasiharmonic approximation, resulting in an effective harmonic Hamiltonian

$$
H=\frac{p^{2}}{2 M}+\frac{1}{2} M \omega_{0}^{2} x^{2},
$$

where the effective phonon frequency is $\omega_{0}$. Then they obtained a nonlinear equation of $\omega_{0}$ :

$$
\left(\frac{\omega_{0}}{\omega_{00}}\right)^{2}=1+\beta \frac{\omega_{00}}{\omega_{0}}\left(\frac{1}{e^{\hbar \omega_{0} / k_{B} T}-1}+\frac{1}{2}-\frac{1}{2} \frac{\omega_{0}}{\omega_{00}}\right)
$$

where $\beta$ represents the magnitude of anharmonicity and $\omega_{00}=\omega_{0}(T=0)$. This equation demonstrates that the effective phonon frequency $\omega_{0}$ has a notable temperature dependence due to the anharmonicity (see Fig. 1 in Ref. 14). Due to the anharmonicity of the phonon mode, the temperature dependence of $1 / T_{1} T$ for the Raman process is qualitatively different from that of harmonic phonons. $1 / T_{1} T \propto T$ at high temperature for harmonic phonons changes to $1 / T_{1} T \sim$ const at high $T$ for anharmonic phonons. They pointed out that the twophonon Raman process dominates NMR relaxation instead of the direct process. The frequency-independent $1 / T_{1} T$ at the La site (see Fig. 1 ) is consistent with the Raman process as studied in Ref. 23. The solid line in Fig. 4 is a calculation in Ref. 14 assuming $\beta=2.0$, $\omega_{0}(T=0)=60 \mathrm{~K}$. The calculation is consistent with the experimental data. Furthermore, the low temperature value of the obtained phonon frequency $\omega_{0}(T=0)=60$ $\mathrm{K}$ is quite consistent with a low Einstein temperature of $59 \mathrm{~K}$ reported in specific-heat experiments, $\frac{3}{-}$ a flat phonon-dispersion energy about $50 \mathrm{~K}$ deduced from the second-order phonon peak related to vibration of the La ions in Raman scattering experiments,,$\frac{5,6}{2,}$ and about 5 $\mathrm{meV}(\sim 60 \mathrm{~K})$ by ab initio calculation ${ }^{24}$ In their calculation, anharmonicity (corresponds to $\beta$ ) plays a crucial role to account for the observed temperature dependence of NMR relaxation. The anharmonicity in the $\mathrm{Os}_{4} \mathrm{Sb}_{12}$ family actually has been observed experimentally from neutron 25,26 and Raman scattering measurements $\stackrel{27}{\stackrel{27}{*} \text { The }}$ value of $\beta=2.0$ in $\mathrm{LaOs}_{4} \mathrm{Sb}_{12}$ is smaller than that of $\mathrm{KOs}_{2} \mathrm{O}_{6}(\beta=6.27)$, suggesting that the anharmonicity of $\mathrm{LaOs}_{4} \mathrm{Sb}_{12}$ is smaller than that of $\mathrm{KOs}_{2} \mathrm{O}_{6}$. This is reasonably understood by the fact that the mass of the $\mathrm{K}$ ion 
is much smaller than that of the La ion. The temperature dependence of the resistivity suggests a stronger anharmonicity in $\mathrm{KO}_{2} \mathrm{O}_{6}$ than in $\mathrm{LaOs}_{4} \mathrm{Sb}_{12}$. A concavedownward temperature dependence is observed in the resistivity in $\mathrm{KO}_{2} \mathrm{Sb}_{12}{ }^{28}$, which is considered to be related to anharmonic phonons, but just a small shoulder is observed in $\mathrm{LaOs}_{4} \mathrm{Sb}_{12} \cdot{ }^{29}$ Therefore, we consider that the additional relaxation at the La site in $\mathrm{LaO}_{4} \mathrm{Sb}_{12}$ originates from the anharmonic phonons due to the rattling motion.

The localized and strongly anharmonic phonon modes of the rare-earth ions observed in $\mathrm{LaOs}_{4} \mathrm{Sb}_{12}$ are presumably a common feature in the $\mathrm{Os}_{4} \mathrm{Sb}_{12}$ cage. We consider that the rattling motion is related to the exotic HF state in $R \mathrm{Os}_{4} \mathrm{Sb}_{12}(R=\mathrm{Pr}, \mathrm{Sm}$, and $\mathrm{Nd})$. In fact, a large mass enhancement is observed in $\mathrm{KO}_{2} \mathrm{O}_{6}$, in which active rattling motion is expected. ${ }^{30}$ If the rattling is related to the formation of the heavy-electron mass, the rattling motion of $f$ electrons at rare-earth sites would be important for the exotic $\mathrm{HF}$ state in $R_{\mathrm{Os}} \mathrm{Sb}_{12}$, because such exotic $\mathrm{HF}$ behavior is not observed in $\mathrm{LaOs}_{4} \mathrm{Sb}_{12}$ without $f$ electrons 29 Very recently, NQR measurements surements on $\mathrm{SmOs}_{4} \mathrm{Sb}_{12}$ have suggested that charge fluctuations originating from the rattling motion of $\mathrm{Sm}$ ions might play an important role in forming the exotic $\mathrm{HF}$ state $^{31}$ Elucidating the relationship between the rattling motion of the $f$ electrons and the exotic HF state is a fascinating problem in $R \mathrm{Os}_{4} \mathrm{Sb}_{12}(R=\mathrm{Pr}, \mathrm{Sm}$, and $\mathrm{Nd})$, which deserves the further experimental and theoretical study.

In conclusion, on the basis of the different temperature dependence of $1 / T_{1} T$ at the La and Sb sites, we found that additional low-lying excitations at the La site exist in $\mathrm{LaOS}_{4} \mathrm{Sb}_{12}$. The temperature dependence of $1 / T_{1} T$ at the La site is consistently understood in terms of the Raman process of anharmonic phonons, attributed to the rattling motion of the La ions. The low-lying excitations caused by the rattling are suggested to be related to the exotic heavy-fermion state in the $\mathrm{ROs}_{4} \mathrm{Sb}_{12}$ family. Further study is required to clarify the relationship.

We thank T. Dahm and M. Takigawa for valuable discussions. Two of the authors (Y.N. and K.I.) thank K. Kitagawa and Y. Maeno for their experimental support. This work was supported by a Grant-in-Aid for the 21st Century COE gCenter for Diversity and Universality in Physicsh from the Ministry of Education, Culture, Sports, Science and Technology (MEXT) of Japan, by Grants-in-Aid for Scientific Research from the Japan Society for the Promotion of Science (JSPS), and by Grantsin-Aid for Scientific Research in Priority Area gSkutteruditeh (Nos. 16037208 and 15072206).
* nakai@scphys.kyoto-u.ac.jp

1 B. C. Sales, D. Mandrus, and R. K. Williams, Science 272, 1325 (1996).

2 V. Keppens, D. Mandrus, B. C. Sales, B. C. Chakoumakos, P. Dai, R. Coldea, M. B. Maple, D. A. Gajewski, E. J. Freeman, and S. Bennington, Nature 395, 876 (1998).

3 H. Harima and K. Takegahara, Physica B 383, 93 (2006).

${ }^{4}$ K. Kaneko, N. Metoki, T. D. Matsuda, and M. Kohgi, J. Phys. Soc. Jpn 75, 034701 (2006).

5 N. Ogita, R. Kojima, Y. Takasu, T. Hasegawa, T. Kondo, M. Udagawa, N. Takeda, T. Ikeno, K. Ishikawa, H. Sugawara, et al., J. Magn. Magn. Mater. 310, 948 (2007).

${ }^{6}$ N. Ogita, T. Kondo, T. Hasegawa, Y. Takasu, M. Udagawa, N. Takeda, K. Ishikawa, H. Sugawara, D. Kikuchi, H. Sato, et al., Physica B 383, 128 (2006).

7 T. Goto, Y. Nemoto, K. Sakai, T. Yamaguchi, M. Akatsu, T. Yanagisawa, H. Hazama, K. Onuki, H. Sugawara, and H. Sato, Phys. Rev. B 69, 180511(R) (2004).

8 E. D. Bauer, N. A. Frederick, P.-C. Ho, V. S. Zapf, and M. B. Maple, Phys. Rev. B 65, 100506(R) (2002).

9 S. Sanada, Y. Aoki, H. Aoki, A. Tsuchiya, D. Kikuchi, H. Sugawara, and H. Sato, J. Phys. Soc. Jpn 74, 246 (2005).

10 H. Sugawara, M. Kobayashi, S. Osaki, S. R. Saha, T. Namiki, Y. Aoki, and H. Sato, Phys. Rev. B 72, 014519 (2005).

11 P.-C. Ho, W. M. Yuhasz, N. P. Butch, N. A. Frederick, T. A. Sayles, J. R. Jeffries, M. B. Maple, J. B. Betts, A. H. Lacerda, P. Rogl, et al., Phys. Rev. B 72, 094410 (2005).

12 K. Hattori, Y. Hirayama, and K. Miyake, J. Phys. Soc. Jpn 74, 3306 (2005).
${ }^{13}$ K. Mitsumoto and Y. Ono, Physica C 426-431, 330 (2005).

14 T. Dahm and K. Ueda, Phys. Rev. Lett. 99, 187003 (2007).

15 H. Sugawara, S. Osaki, S. R. Saha, Y. Aoki, H. Sato, Y. Inada, H. Shishido, R. Settai, Y. Ōnuki, H. Harima, et al., Phys. Rev. B 66, 220504(R) (2002).

16 M. Yogi, T. Nagai, Y. Imamura, H. Mukuda, Y. Kitaoka, D. Kikuchi, H. Sugawara, Y. Aoki, H. Sato, and H. Harima, J. Phys. Soc. Jpn 75, 124702 (2006).

17 H. Kotegawa, M. Yogi, Y. Imamura, Y. Kawasaki, G.-q. Zheng, Y. Kitaoka, S. Ohsaki, H. Sugawara, Y. Aoki, and H. Sato, Phys. Rev. Lett. 90, 027001 (2003).

${ }^{18}$ Y. Nakai, K. Ishida, D. Kikuchi, H. Sugawara, and H. Sato, J. Phys. Soc. Jpn 74, 3370 (2005).

19 Y. Nakai, K. Ishida, K. Magishi, H. Sugawara, D. Kikuchi, and H. Sato, J. Magn. Magn. Mater. 310, 255 (2007).

20 H. Harima and K. Takegahara, Physica C 388-389, 555 (2003).

21 Y. Nemoto, private communications.

22 M. Yoshida, K. Arai, R. Kaido, M. Takigawa, S. Yonezawa, Y. Muraoka, and Z. Hiroi, Physical Review Letters 98, 197002 (2007).

23 J. V. Kranendonk, Physica 205, 781 (1954).

24 T. Hasegawa, private communications.

${ }^{25}$ K. Iwasa, M. Kohgi, H. Sugawara, and H. Sato, Physica B 378-380, 194 (2006).

26 K. Kaneko, private communications.

27 M. Udagawa, private communications.

28 Z. Hiroi, S. Yonezawa, J. Yamamura, T. Muramatsu, and Y. Muraoka, J. Phys. Soc. Jpn 75, 1682 (2005).

${ }^{29}$ H. Sugawara, M. Kobayashi, S. Osaki, S. R. Saha, 
T. Namiki, Y. Aoki, and H. Sato, Phys. Rev. B 72, 014519 (2005).

30 M. Bruhwiler, S. M. Kazakov, J. Karpinski, and B. Batlogg, Physical Review B (Condensed Matter and Materials Physics) 73, 094518 (2006).
${ }^{31}$ H. Kotegawa, H. Hidaka, T. C. Kobayashi, D. Kikuchi, H. Sugawara, and H. Sato, Phys. Rev. Lett. 99, 156408 (2007). 